\section{THU0512 WHAT IS HIDDEN BEHIND SYSTEMIC JUVENILE IDIOPATHIC ARTHRITIS, ADULT ONSET STILL'S DISEASE AND SECONDARY MACROPHAGIC ACTIVATION SYNDROME. THE UTILITY OF THE BIOMARKERS}

Alina Boteanu ${ }^{1}$, Carlota García-Hoz ${ }^{2}$, Andrea Briones-Figueroa ${ }^{2}$, Daniel Clemente ${ }^{3}$, Juan Carlos Nieto Gonzalez ${ }^{4}$, Martina Steiner ${ }^{5}$, Concepción Morado Quiroga ${ }^{6}$, Antía García Fernández ${ }^{2}$, Maria Luz Gámir Gámir², Monica Vazquez Diaz ${ }^{2}$. University Hospital Ramón y Cajal, Rheumatology and Pediatric Rheumatology Unit, Madrid, Spain; ${ }^{2}$ University Hospital Ramón y Cajal, Madrid, Spain; ${ }^{3}$ University Hospital Niño Jesus, Madrid, Spain; ${ }^{4}$ University Hospital Gregorio Marañon, Madrid, Spain; ${ }^{5}$ University Hospital Infanta Sofia, Madrid, Spain; ${ }^{6}$ UNiversity Hospital Clinico San Carlos, Madrid, Spain

Background: Systemic Juvenile Idiopathic Arthritis (SJIA) and adult onset Still's disease are rare diseases of unknown etiology that share several clinical and laboratory features. The pathogenesis of these two diseases is complex and multifactorial, with an important role of innate immunity: activation of neutrophils and macrophages and increased levels of cytokines dependent on the activation of the inflammosome. A proportion of these patients will develop a Macrophage activation syndrome (MAS), a serious and life-threatening complication.

Objectives: To identify immunological markers that allow a differential diagnosis between patients with inactive SJIA, active SJIA or MAS. To evaluate if there are differences between biomarkers in SJIA and adult onset Still's disease

Methods: Observational, prospective and multicenter study. Inclusion criteria: patients with SJIA or adult onset Still's disease, followed in Paediatric or Adult Rheumatology Department from 5 Madrid hospitals. Group 1: Patients with active SJIA or adult onset Still's disease +/- diagnosis of secondary MAS at the first visit, have been followed-up performing a second clinical and analytical visit at 3 months. Group 2: Patients with inactive SJIA or adult onset Still's. Clinical and analytical data were collected, PBMCs were obtained before and after treatment, and several leukocyte subtypes were evaluated by flow cytometry (FACS). The intracytoplasmic expression of TNF- $\alpha, \mathrm{IL}-1 \mathrm{~b}$ and IL- 6 in monocytes was determined by flow cytometry before and after stimulation with lipopolysaccharides (LPS) in the presence of monensin (Golgi inhibitor). In addition, IL-18 levels were determined by ELISA in all visits.

Results: A total of 25 patients were included (18 SJIA and 7 adult onset Still's diseases), 2 of them being excluded from the analysis because exclusions criteria. $30,4 \%$ patients presented MAS at the first visit (5 SJIA and 2 adult onset Still's disease) and other 2 at some time during the disease. The median of IL-18 was 296.1 (Cl 108.3-346.0) in patients who have never had MAS and 899.85 (Cl: 397.32-1095.6) in the other group. Patients with active MAS had a CD4/CD8 ratio close to the lower limit $(1.1+/-0.1 \mathrm{SD})$ reflecting a decrease in the CD4 + population and an increase in CD8 +. On the other hand, the rest of the groups presented a CD4/CD8 ratio of $2.6+/-0.5 \mathrm{SD}$ in patients without disease activity and $2.0+/-0.7$ SD in patients with active SJIA or adult onset Still's disease but without MAS. No significant differences were found between patients with SJIA or adult onset Still's disease.

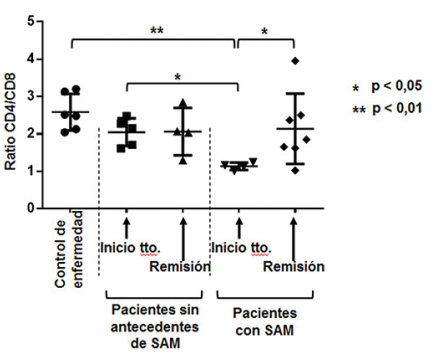

Abstract THU0512 - Figure 1

Conclusion: The results of this study show that the analysis of three determinations:\%CD4,\%CD8 and the CD4/CD8 ratio can help in the early detection of patients with MAS secondary to SJIA or adult onset Still's disease. The determination of IL-18 has been in our study a marker of MAS, similar to several published studies. No significant differences were found in the immunological tests between patients with SJIA and adult onset Still's disease with active or inactive disease.

\section{REFERENCE:}

[1] Weiss ES et all Interleukin-18 diagnostically distinguishes and pathogenically promotes human and murine macrophage activation syndrome. Blood. 2018 Mar 29;131(13):1442-1455

Disclosure of Interests: None declared

DOI: 10.1136/annrheumdis-2019-eular.6355

\section{THU0513 WHOLE BLOOD CELLS FROM PATIENTS WITH SYSTEMIC JUVENILE IDIOPATHIC ARTHRITIS (SJIA) IN CLINICAL INACTIVE DISEASE DISPLAY A DYSREGULATED RESPONSE TO TLR-4 STIMULATION}

Antonia Pascarella, Manuela Pardeo, Ivan Caiello, Claudia Bracaglia, Marianna Rossi, Giulia Marucci, Emanuela Sacco, Fabrizio De Benedetti, Giusi Prencipe. Bambino Gesù Children's Hospital, IRCCS, Rome, Italy

Background: Systemic juvenile idiopathic arthritis (SJIA) is a polygenic autoinflammatory disease. Innate immune mechanisms appear to play a central role in the pathogenesis of the disease. Nevertheless, a better understanding of the pathophysiology of SJIA is still needed to identify patients responsive to IL-1 or IL- 6 targeted therapies.

Objectives: In this study, we evaluated the production of IL-1 $1 \beta, \mathrm{IL}-6$ and TNF- $\alpha$ by fresh whole blood cells isolated from SJIA patients in disease remission, after stimulation with the TLR-4 ligand lipopolysaccharide (LPS), and we investigated whether SJIA patients that respond or notrespond to treatment with the IL-1 receptor antagonist anakinra show a different response.

Methods: We collected fresh whole blood samples from sJIA patients during clinical inactive disease (inactive $\mathrm{SJIA}, \mathrm{n}=19$ ) and $\mathrm{SJIA}$ patients during active disease (active sJIA $n=4$ ). Active and inactive disease was defined at time of sampling. As controls, fresh whole blood samples from healthy subjects (HS, n=10) were used. Whole blood cells were left unstimulated or stimulated with $10 \mathrm{ug} / \mathrm{mL}$ of LPS for 24 hours. Cytokine levels (IL-1 $\beta$, IL- 6 and TNF- $\alpha$ ) released in the supernatants were measured by ELISA Response to anakinra was defined as achievement of clinical inactive disease off glucocorticoids at 6 months after initiation of anakinra treatment. Results: We found that LPS-stimulated cells from inactive SJIA patients released significantly higher amounts of all the inflammatory cytokines tested, compared to HS $(p<0.01)$. In addition, cells from inactive sJIA patients produced significantly higher levels of IL-1 $\beta$ also compared to active sJIA patients $(p<0.05)$. When we divided inactive sJIA patients in two groups (responders and non-responders), depending on the clinical response to anakinra treatment, we observed that in both groups of patients IL-1 $1 \beta$, IL- 6 and TNF- $\alpha$ levels were significantly higher than those observed in HS. In addition, we found that LPS-stimulated whole blood cells from non-responder inactive SJIA patients released significantly higher levels of IL-1 $\beta$ and TNF- $\alpha$ compared to responder inactive SJIA patients.

Conclusion: Our preliminary results show a dysregulated production of inflammatory cytokines by whole blood cells from sJIA patients in remission disease, when stimulated with a TLR-4 agonist.

Disclosure of Interests: : Antonia Pascarella: None declared, Manuela Pardeo: None declared, Ivan Caiello: None declared, Claudia Bracaglia: None declared, Marianna Rossi: None declared, Giulia Marucci: None declared, Emanuela Sacco: None declared, Fabrizio De Benedetti Grant/ research support from: Abbvie, SOBI, Novimmune, Roche, Novartis, Sanofi, Pfizer, Giusi Prencipe: None declared

DOI: 10.1136/annrheumdis-2019-eular.6895

\section{THU0514 ROLE OF THE ORAL MICROBIOME IN CHRONIC NON-} BACTERIAL OSTEOMYELITIS IN CHILDREN

Mona Zeus, Stefan Janssen, Ute Fischer, Hans Jürgen Laws, Arndt Borkhardt, Prasad Oommen. University Duesseldorf, Duesseldorf, Germany

Background: Chronic non-bacterial osteomyelitis (CNO) is a rare autoinflammatory disease of the bone and bone marrow. The presentation of the disease is broad, but pain and osteoedema on MRI are the hallmarks of CNO. While the activation of inflammasomes and the subsequent release of IL-1B seems to play a central role, the exact molecular pathophysiology of $\mathrm{CNO}$ is largely unexplained. In respect of the potential role of the microbiome to date only experimental data on mice are available, indicating that the manipulation of the microbiome through dietary modulation might alter the course of the disease and also plays a role in the release of IL-1B from innate immune cells ${ }^{1}$. 